\title{
Comments
}

\section{Great Mistake of Great J. C. Maxwell}

\author{
Robert A. Sizov ${ }^{1 *}$ \\ ${ }^{1}$ Doctor of physics and mathematics, Individual researcher, Moscow, Russia
}

Received: January 9, 2020

Accepted: January 20, 2020

Online Published: January 30, 2020

doi:10.22158/asir.v4n1p32

URL: http://dx.doi.org/10.22158/asir.v4n1p32

\begin{abstract}
The Maxwell's vicious electromagnetic concept which can also be defined as electric magnetism occurred as a result of superficial and, exclusively, erroneous impression of the Great Theorist on the famous Oersted Experience. The fateful concept for physical science: "electrons moving in a conductor are direct sources of a magnetic field", was put forward by Maxwell in the absence of knowledge of real physical processes occurring in a conductor with an electric current. Paradoxical as it may see but this vicious concept, again thanks to the outward impression, has been accepted by the world physical community and dominates in physical science for almost 150 years, as an absolute truth. As the result the true sources of the magnetic field - real magnetic poles (magnetic charges) which are the structural components of atoms and substance, were "buried alive". It is Maxwell's vicious electric magnetism that suppressed the interest of scientists in the search for and detection of real magnetic charges in atoms and substance and pushed of the physical theory into the "swamp" of relativism. The author's experimental and theoretical studies have shown that a main reason for ignoring magnetic charges and, and therefore, the emergence of a vicious concept of electric magnetism, is the radically different physics of the confinement of electrons and magnetic charges in the structures of Physical mass (in atoms, nucleons, substance and others).
\end{abstract}

\section{Keywords}

Electrons, magnetic charges (magneton and antimagneton), vortex electromagnetic (gravitational) field, magnetic moments, Dirac monopole, Maxwell's electric magnetism

\section{Introduction}

The main reason that has delayed the official recognition of real magnetic charges, as well as true antielectrons by almost 150 years is the physics of its confinement in atoms and substance radically different from of confinement electrons. In addition, a very significant negative factor in the problem with the recognition of real magnetic charges was the vicious concept of Maxwell's electric magnetism (Maxwell, 1873). Within the framework of this concept the great physicist, in the absence of real 
knowledge physical processes occurring in a conductor with electric current and guided solely by the superficial impression of the famous Oersted Experience, "deprived" the magnetic field of its own source, i.e., of the magnetic pole or magnetic charge. Surprisingly, this erroneous concept was perceived by the world physical community and dominates physical science for almost a century and a half as absolute truth. In many ways, "thanks" to this of the vicious electromagnetic concept the magnetic charges were "buried alive", and the magnetic field emitted by them turned into of "miscarriage" of electricity.

As a result of more than a century of dominance of vicious electric magnetism in physical science, the "Himalayas" of theoretical "discoveries" were built that were not related to reality. Among these "discoveries" we can name: the curvature of 4-dimensional space, as an explanation of gravity, the global expansion of the Universe, the big bang, the fantastic travels through "Black holes", annihilation of fundamental particles in pair the particle-antiparticle and so many others.

However, most harmful "exhaust" of Maxwell's vicious concept is the model version of purely electron shell of atoms. This vicious version backed by works of E. Rutherford and N. Bohr, arose solely as a result ignoring in the physical theory of the existence of real magnetic charges in atomic structures. The concept of electron shell of atoms as the "gravestone" closed all possibilities of approximation science to real atomic physics, as well as to the physics of the gravitational field. For the same reason, the physical nature of the Physical Mass remained beyond real knowledge that and determined the appearance of such a famous theoretical surrogate as the H-boson, which is fictitious, exclusively mathematical source of mass. The vicious concept of the electron shell of atoms provoked the formation of erroneous ideas and in related sciences, for example, in chemistry, in the form of an erroneous electronic theory of chemical bonding.

The list of especially significant erroneous provisions in existing physical theory, generated by vicious concept of Maxwell is presented in the author's publication (Robert, 2019). However, the global harmfulness of this concept is not limited to erroneous interpretations, on its basis, of effects and manifestations. The vicious electric magnetism has blocked the possibilities of detecting and putting into practice new useful effects and manifestations, among which, for example, are such the practically important effect discovered by the author of the article as gravitational levitation (Robert, 2019). A list of useful innovations discovered by the author when introducing real magnetic charges into basic physical concepts is given in Robert (2015).

The discovery and many years of research by the author of real magnetic charges, as well as true antielectrons in atoms and substance (period 1970 - present) have shown that atomic shells are electromagnetic, and not electronic (Robert, 1971, 2001, 2008, 2015, 2016). In addition to electrons, three more fundamental particles function in their compositions: two magnetic particles (a magneton and an anti-magneton respectively with magnetic charges $\mathrm{g}^{-}$and $\mathrm{g}^{+}$), as well as a true anti-electron with a charge $\mathrm{e}^{+}$. The charges of all particles in the compositions of atomic shells correspond to the relation $\mathrm{e}$ $=\mathrm{g}$, where $\mathrm{e}$ is the electron charge. It is the electromagnetic shells are the sources of the vortex 
electromagnetic (gravitational) field.

In Figure 1 in the framework of the structure of the atom of so-called light hydrogen (protium), atomic shells are shown: universally recognized in present purely electronic shell (1a) and existing in reality electromagnetic shell (1b). The electromagnetic shell of a hydrogen atom consists of two coupled dipoles (electric and magnetic) which in antiphase rotate in the same atomic orbit. This electromagnetic complex of four spinor particles, received the author's name S-Graviton (S from word a source), is an elementary source of the vortex electromagnetic (gravitational) field which is described by the vortex vector $\operatorname{rot}[\mathbf{E}-\mathbf{H}]$, where $\mathbf{E}$ and $\mathbf{H}$ are vectors instantaneous strength of electric and magnetic fields in the composition of the vortex electromagnetic (gravitational) field.
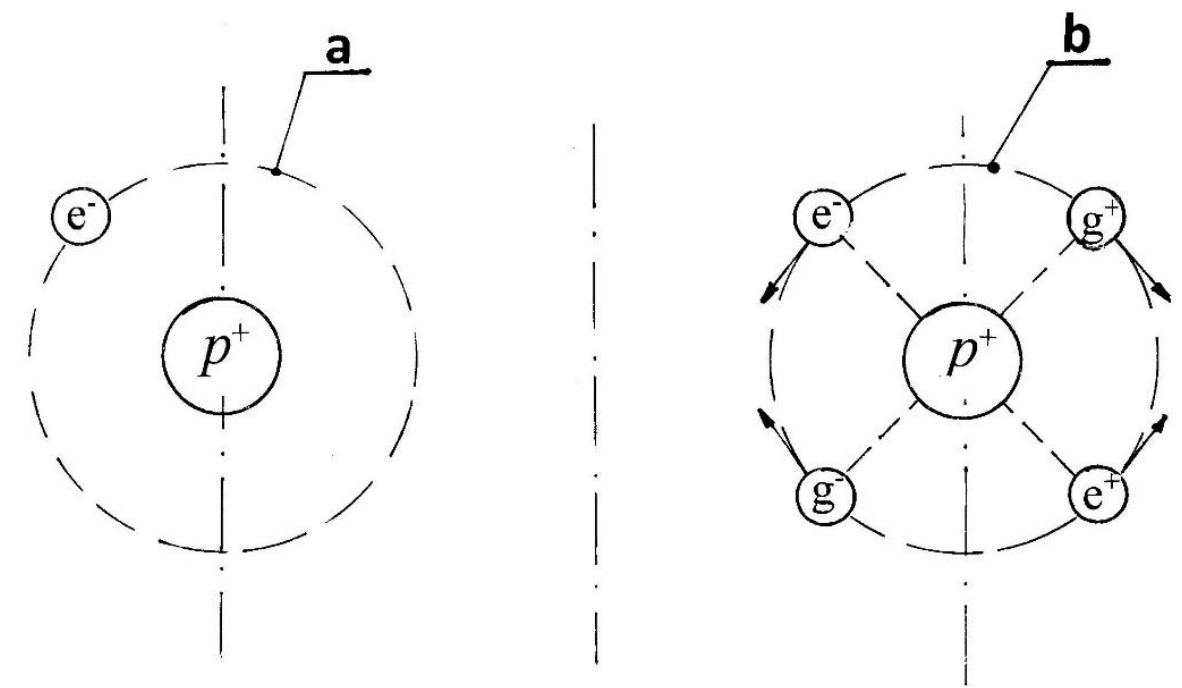

Figure 1. Schematic Diagrams of the Structure of a Hydrogen Atom (Protium): The Currently

\section{Generally Accepted Atom with a Purely Electronic Shell (1a) and the Atom Existing in Reality}

\section{with an Electromagnetic Shell (1b)}

At the center of the atoms is the nucleus, which is the proton $\left(p^{+}\right)$with the charge $\mathrm{e}^{+}$. Arrows for electric and magnetic charges in Figure 1b indicate the direction of rotation of the electric and magnetic dipoles in composition of electromagnetic shell of the protium atom.

The equation of the process of generation of the gravitational field by S-Graviton is written in the form: $\mathrm{k} \operatorname{rot}\left[\mathbf{J}_{\mathrm{e}}-\mathbf{J}_{\mathrm{g}}\right]=\operatorname{rot}[\mathbf{E}-\mathbf{H}]$, where $\mathbf{J}_{\mathrm{e}}$ and $\mathbf{J}_{\mathrm{g}}$ are the vectors of the instantaneous density of the orbital currents of electric (e) and magnetic ( $\mathrm{g}$ ) charges, and $\mathrm{k}$ is coefficient of proportionality. The minus signs, indicated in the above equation for formation of the gravitational field correspond to both the antiphase of the orbital (dipole) currents of electric and magnetic charges, and the anti-directionality of the vectors of the instantaneous electric and magnetic fields strength at of each point of gravitational field.

It should be recognized that ignoring the three real fundamental particles, i.e., magnetic charges and true antielectrons in the structures of atoms and substance could not pass without a trace for physical 
theory. These unrecognized fundamental particles were forcedly replaced by known theoretical surrogates: magnetic charges were replaced by magnetic moments, and the role of true antielectrons was assigned to electronic vacancies or Dirac holes. Of course, the noted surrogates could not fully replace the real fundamental particles which and determined the extremely complex state of modern physical theory.

In his publications the author constantly emphasized that the main reason for the appearance of Maxwell's electric magnetism is the selective physics of the confinement of electric and magnetic spinor particles in the structures of the mass (atoms, nucleons, substance, etc.) (Robert, 2015, 2017).

Figure 2 makes it possible to clarify the essence the selectivity while confinements of electrical and magnetic particles in the structures of substance. In Figure 2 shows the scheme disposition of levels electrons, magnetic charges and true antielectrons in of the condensed state substance on the energy scale. It is well known that the electronic levels are located in the potential pit and for going out of an electron in a free state is necessary to increase the internal energy of substance, for example, heat it.

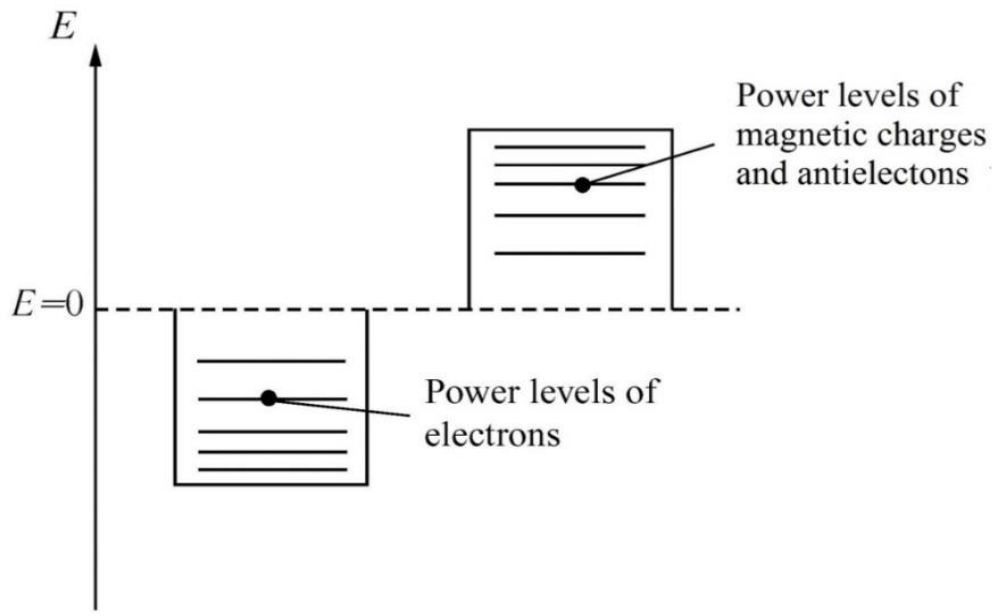

Figure 2. The Location on Scale of Energy (in the Condensed State of Substance) Levels of Electrons (in the Potential "Pit") and Magnetic Charges with True Antielectrons (under the Potential "Cupola")

Unlike electrons, the energy levels of true antielectrons, as and the levels of magnetic charges are under potential cupola and for the implementation of the free state of these particles need to cooled substance, that is, to reduce its internal energy. The deep stages of cooling there is arises the superconductivity effect what is an evidence of transition part of the magnetic charges, as and antielectrons into the condition free from bonds with the lattice of superconductor.

It is also useful to note that the orientation of modern fundamental physics to high-energy projects appears to the author to be a kind of "stop-cock" that closes the possibility of approximation to real magnetic charges. In his publications the author showed that the range of energies in which it is possible to carry out controlled practical activities with individual magnetic charges and their currents 
is the ultra-low temperatures.

The first person to experimentally observe real magnetic charges was the remarkable Austrian physicist Felix Ehrenhaft, who for more than 40 years (1910-1952) explored these "elusive" fundamental particles that actually exist in atoms and substance (Ehrenhaft, 1951). The essence of his experiments consisted in observing the motion of small particles of various substances ionized by magnetic charges of different signs in a vertical magnetic field. These movements activated when particles were irradiated with a concentrated beam of light. The minimum magnetic charge detected on the particles was close to the magnitude of the electron charge. However, the interpretation of the results experiments, which was constructed by Ehrenhaft as analogy with the behavior of electric particles in an electrostatic field is not convincing. In addition, the forces in observed interactions were comparable with the so-called spurious radiometric forces. We also note that such serious conclusions that were made by Ehrenhaft needed more serious development and, above all, in terms of idea about the place of magnetic charges in the structures of substance. Apparently in this is the reasons that works of Ehrenhaft and his many followers did not receive recognition and is now practical forgotten.

As is known, in the framework of the concept electric magnetism a formation of vortex magnetic field rotH by an electric current is described by the so-called the first Maxwell equation: $\mathrm{k} \mathbf{J}_{\mathrm{e}}=\operatorname{rot} \mathbf{H}$, where $\mathrm{J}_{\mathrm{e}}$ is the density vector of the linear electric current in the conductor, and $\mathrm{k}$ is the proportionality coefficient. However, the physical process itself of formation of this magnetic field by the moving electric charges remains the biggest mystery of physical science for almost 150 years. Modern theory attributes all this to very confusing theoretical surrogate, the so-called relativistic effect. The introduction of real magnetic charges into basic physics allows us to simply and naturally explain the formation of a magnetic field. The fact is that electrons in the process of their movement act on the magnetic charges that exist in the conductor in composition of magnetic dipoles and bring these dipoles into circular (vortex) motion. This process is described by the equation $\mathrm{k}_{1} \mathbf{J}_{\mathrm{e}}=\operatorname{rot} \mathbf{J}_{\mathrm{g}}$, where $\mathbf{J}_{\mathrm{g}}$ is the vector of instantaneous current density of magnetic charges in the composition of magnetic dipole vortex. In the next process, rotating magnetic dipoles create a known vortex magnetic field rotH around the conductor according to the equation $\mathrm{k}_{2} \operatorname{rot} \mathrm{J}_{\mathrm{g}}=\operatorname{rot} \mathbf{H}$. Thus, in the framework of the first Maxwell equation, not one physical process is realized, as the Great Physicist seemed, but the two shown above. Electrons in the process of formation of a magnetic field are exclusively intermediary who in the process of itself movement engaged, exclusively, of untwisting magnetic dipoles. As for the magnetic field, its sources, as it should be in Nature, are magnetic poles (magnetic charges).

Now we can confidently state that practically none of the scientists who were active in physics after Maxwell, did not escape the infection by of Maxwell's vicious "virus" (the definition of Maxwell's electric magnetism that given by the author of this article). But most striking fact is that himself ideologist of magnetic monopolism P. Dirac did not doubt in justice of doctrine Maxwell's (Dirac, 1931). Viewpoint that he took as regards on issues associated with magnetic poles is represented more than strange. On the one hand, Dirac proclaim that it would be surprising if Nature would not have 
utilized this possibility, i.e., that of realizing magnetic poles in structures of substance, and he even showed that the quantum electromagnetic theory quite allows them existence. But on the other side, he is filled his monopole with a magnetic field, which was produced in ultrathin filaments-solenoid fed by an electric current. Thus, if to remove the Dirac's solenoids, i.e., if disconnect monopole from the electric current source then there will be no and famous monopole. In other words, despite high goals by Dirac and his magnetic monopole not failed to avoid "infection" of Maxwell's vicious "virus" (Robert, 2018).

In addition, the magnetic field emitted by the Dirac monopole can be only a vortex field, which is described by the vortex vector rotH since only such a magnetic field can be formed by means of an electric current. It is also important to note that Dirac did not associate his monopole with magnetic fields and manifestations which surround man, because he did not see any prospects for this.

\section{Conclusion}

The "pathological" situation that has developed in fundamental physical theory in connection with the domination in it, almost 150 years, of Maxwell's vicious conception in it strikes a normal imagination. It is exclusively under the influence of this concept the world physical theory has ignored of the existence in Nature, practically, of three quarters of fundamental spinor particles, has leaving yourself only electric and neutral particles for "convenience" of physical interpretations. An indicative example of such electro-magnetic scenario is the physically vicious but nevertheless generally accepted in physical science the electron shells of atoms.

The vicious electromagnetic concept of Maxwell, given its general-physical harmfulness, can to associate with the "cancerous formation" in the "body" of physical science, the "metastases" of which have hit its many areas and directions. Conducting global "chemotherapy" of a modern physical theory, with the purpose of eliminating the numerous misconceptions generated by Maxwell's vicious concept, according to the author, is the most urgent task for today. All this needs to be done, and as soon as possible because after 10 - 15 years even robots will laugh at us.

\section{Acknowledgments}

The author expresses deep gratitude to Elena Sizova and Vitaly Sizov who for many years helped and supported him which largely contributed to the implementation of his research activities.

\section{References}

Dirac, P. A. M. (1931). Quantized Singularities in the Electromagnetic Field. Proceedings of the Royal Society, A133, 60 .

Ehrenhaft, F. (1951). On Photophoresis, the true magnetic Charge and on helical Motion of Matter in Fields (Review of his scientific work part 1, in German). Acta Physica Austriaca, 4, 461-488.

Maxwell, J. C. (1873). Treatise on Electricity and Magnetism (Vol. 1-2). Clarendon Press, Oxford. 
Robert A. Sizov. (1971). Delocalization of the magnetic moment of $\mathrm{Fe}^{3+}$ ions in $\mathrm{Y}$ type hexagonal ferrite at $293^{\circ} \mathrm{K}$. Journal of Experimental and Theoretical Physics, 60, 1363-1370.

Robert A. Sizov. (2001). New Presentation of Nature Magnetism, Gravitation and Nuclear Forces of Bonding. Akademizdat Center "Science", Moscow.

Robert A. Sizov. (2008). Electric and Magnetic Spinor Particles as Structure-Forming Components of Mass and Electromagnetic Source Gravitation. Akademizdat Center "Science”, Moscow.

Robert A. Sizov. (2015) Real Magnetic Charges in the Substance, Ferrogravitation and Technical Levitation. Journal of Modern Physics, 6, 1591-1601. http://doi.org/10.4236/jmp.2015.611161

Robert A. Sizov. (2015). Dirac's "holes" are the true Antielectrons and real particles of Antimatter. Journal of Modern Physics, 6(15), 2280-2289. http://doi.org/10.4236/jmp.2015.615232

Robert A. Sizov. (2015). Real magnetic poles (magnetic charges) in the Physics of magnetism, gravitation and levitation. Journal of Modern Physics, 6(8), 1013-1022. http://doi.org/10.4236/jmp.2015.68106

Robert A. Sizov. (2016). Electromagnetic Shells of Atoms and Periodic System of Elements. Journal of Modern Physics, 7(16), 2374-2397. http://doi.org/10.4236/jmp.2016.716206

Robert A. Sizov. (2017). "Electrical" and "Magnetic" Worlds in Universe. Journal of Modern Physics, 8(7), 1072-1086. http://doi.org/10.4236/jmp.2017.87069

Robert A. Sizov. (2018). Real Magnetic Charges as A Negation of the Electric Magnetism Maxwell and Electrified Dirac's Monopole. Phys Astron Int J., 2(1), 17-20. http://doi.org/10.15406/paij.2018.02.00043

Robert A. Sizov. (2019). The "naked electron”. Applied Science and Innovative Research, 3(4), 257-263. http://doi.org/10.22158/asir.v3n4p257

Robert A. Sizov. (2019). The electrical magnetism of Maxwell (1873) is the "crooked mirror" of physical science. Applied Physics Research, 11(3), 49-55. http://doi.org/10.5539/apr.v11n3p49_ 